\title{
Linking lignocellulosic dietary patterns
} with gut microbial Enterotypes of Tsaitermes ampliceps and comparison with Mironasutitermes shangchengensis

\author{
L.-J. Su', Y.-Q. Liu', H. Liu', Y. Wang ${ }^{1}$, Y. Li ${ }^{1}$, H.-M. Lin², F.-Q. Wang ${ }^{1,3}$ \\ and A.-D. Song ${ }^{1,3}$ \\ ${ }^{1}$ College of Life Sciences, Henan Agricultural University, Zhengzhou, Henan, China \\ ${ }^{2}$ Termites Prevention Center in Macheng in Hubei, Macheng, Hubei, China \\ ${ }^{3}$ Key Laboratory of Enzyme Engineering of Agricultural Microbiology, \\ Ministry of Agriculture, Zhengzhou, Henan, China \\ Corresponding author: A.-D. Song \\ E-mail: song1666@126.com / sulijuan816@126.com
}

Genet. Mol. Res. 14 (4): 13954-13967 (2015)

Received January 30, 2015

Accepted June 26, 2015

Published October 29, 2015

DOI http://dx.doi.org/10.4238/2015.October.29.16

ABSTRACT. Tsaitermes ampliceps (lower termites) and Mironasutitermes shangchengensis (higher termites) are highly eusocial insects that thrive on recalcitrant lignocellulosic diets through nutritional symbioses with gut dwelling prokaryotes and eukaryotes. We used denaturing gradient gel electrophoresis and a 16S rRNA clone library to investigate i) how microbial communities adapt to lignocellulosic diets with different cellulose and lignin content, ii) the differences in the dominant gut microbial communities of the 2 types of termites. The results indicated that gut microbiota composition in $T$. ampliceps was profoundly affected by 2 -week diet shifts. Comparison of these changes indicated that Bacteroidetes and Spirochaetes act in cellulose degradation, while Firmicutes were responsible for lignin degradation. Additionally, Proteobacteria consistently participated in energy production and balanced the gut environment. Bacteroidetes may 
function without hindgut protozoans in higher termites. The diversity of enteric microorganisms in $M$. shangchengensis was higher than that in $T$. ampliceps, possibly because of the more complicated survival mechanisms of higher termites.

Key words: Denaturing gradient gel electrophoresis; Gut microbiota; Lignocellulosic diets; Mironasutitermes shangchengensis;

Tsaitermes ampliceps

\section{INTRODUCTION}

Colonies of termites are canonical examples of highly eusocial insects, and their success, adaptability, diversity, and eusociality are thus inextricably linked with the composition, diversity, and digestive capabilities of their gut symbionts (Scharf et al., 2011; Brune, 2014). The foregut of lower termite is relatively simple, while the expansion portion or paunch stomach of the hindgut is filled with a large number of flagellates (protozoa). However, higher termites have a highly partitioned gut containing a very small amount of intestinal protozoa. Several different morphological types and densities of bacteria and archaea settle in the digestive tract of both types of termites; particularly, the number of bacteria in the hindgut can reach up to $10^{5}-10^{11} / \mathrm{mL}$. It was estimated that a single Reticulitermes flavipes hindgut contained at least $3 \times 10^{6}$ heterotrophs according to anaerobic cultivation and direct counting methods (Schultz and Breznak, 1978). These symbiotic microorganisms can break down plant fiber and ferment the products to acetate and variable amounts of methane, with hydrogen as a central intermediate; termites thus rely on the biosynthetic capacities of their gut microbiota as a nutritional resource (Yang et al., 2005; Matson et al., 2010; Brune, 2014).

Termites play an important role in the degradation of dead plant materials in nature. Over the last century, numerous studies have investigated the mechanisms of their lignocellulosedigesting system. Several previous studies examining the intestinal microbial community were based mainly on the use of anaerobic culture techniques, which are typically very labor-intensive and time-consuming. However, this estimate did not include the unculturable bacteria associated with the protozoan community, which now is recognized as a major component of the termite intestinal microbial community (lida et al., 2000; Dolan, 2001; Stingl et al., 2005; Ohkuma et al., 2007). The diversity and complexity of the termite gut microbiota have been evaluated using molecular technology techniques based on 16S rRNA (Warnecke et al., 2007; Berlanga et al., 2011; Mathew et al., 2012). Recent studies have indicated that different dietary habits shape the structure of microbiota in the termite gut (Boucias et al., 2013; Huang et al., 2013; Raychoudhury et al., 2013). Dietary manipulation induces measurable differences among gut bacterial communities, and the recalcitrance of plant substrates may be the major cause of this variation in the diversity and richness of gut bacterial communities (Boucias et al., 2013; Huang et al., 2013; Raychoudhury et al., 2013). However, influences on the abundance and diversity of the intestinal microbial of termite fed with different substrates containing various lignin and cellulose or hemicellulose remain unclear.

Tsaitermes ampliceps is a canonical example of lower termites of Rhinotermitidae, while Mironasutitermes shangchengensis is a type of higher termite and are only widely distributed 
in the Henan Province in China (Su et al., 2011). For the insects that cause significant damage to forests and released by the Chinese Ministry of Forestry, the taxonomic composition of the gut prokaryotic microbiota and its relative role(s) in lignocellulose degradation in the 2 types of termites have not been determined. To comprehensively understand the distinction between the 2 types of termites and changes in the dominant gut microbial communities in the lower termites $T$. ampliceps fed with 3 types of food (wood, corn stalk, and filter paper) with different cellulose, hemicellulose, and lignin content, we used 2 different molecular techniques, including denaturing gradient gel electrophoresis (DGGE) analysis and a 16S rRNA clone library to explore these issues.

\section{MATERIAL AND METHODS}

\section{Termite collection and experimental design}

T. ampliceps was collected in May 2012 from the town of Jiushan in Xuchang, located at $\mathrm{N} 34.22^{\circ} \mathrm{E} 113.18^{\circ}$, while $M$. shangchengensis was collected in October 2011 from the town of Jingangtai in Xinyang, located at $\mathrm{N} 31.8^{\circ} \mathrm{E} 115.5^{\circ}$. They were fed in complete darkness at $25^{\circ} \mathrm{C}$ and $>70 \%$ humidity until they were used in the experiment.

$T$. ampliceps collected from the same colony was divided into 3 groups and fed with Robinia pseudoacacia, corn stalk, and filter paper, with the following compositions: i) R. pseudoacacia (W) containing approximately $25-30 \%$ lignin and $50-60 \%$ cellulose and hemicellulose; ii) corn stalk (C) containing approximately $10-15 \%$ lignin and $55-65 \%$ cellulose and hemicellulose; iii) filter paper (F) containing only cellulose. All processing conditions were the same among groups except for the 2 weeks of different diets. Diet materials were cut into approximately $0.5-\mathrm{cm}$ pieces and oven-dried before use. All feeding boxes were $10 \times 10 \times 10 \mathrm{~cm}$ and prepared using $500 \mathrm{~mL} 1 \%$ agarose as basal and moderate one of diet assays on the basal. Boxes were sealed with breathable cotton gauze and placed in a dark incubator at $25^{\circ} \mathrm{C}$ for 2 weeks; each diet treatment was rehydrated with $100 \mathrm{~mL}$ water after every $48 \mathrm{~h}$.

\section{Extraction of DNA from gut microbes and polymerase chain reaction (PCR) amplification}

Fifty workers were dissected in an ice-cold dish with their intestinal tracts removed using a sterile needle from the abdominal tips. Tracts were placed in $800 \mu \mathrm{L}$ ice-cold $0.2 \mathrm{M}$ phosphate-buffered saline, $\mathrm{pH}$ 7.4. The intestinal tracts were disrupted using Bullet Blender ${ }^{\mathrm{TM}}$ (Next Advance, Averill Park, NY, USA). After centrifugation at $200 \mathrm{~g}$ at $4^{\circ} \mathrm{C}$ for $3 \mathrm{~min}$, the supernatant was removed. The total genomic DNA of gut microbes was isolated from the samples using the Soil DNA Kit (Omega Bio-Tek, Inc., Norcross, GA, USA) according to the manufacturer protocol.

Meta-genomic DNA was used as a template for amplification after proper dilution in a LabCycler Standard PCR (SensoQuest, Göttingen, Germany). The V3 region of the 16S rRNA gene was amplified using the eubacteria-specific primers F341-GC and R518 (F341-GC, 5'-CGC CCG CCG CGC GCG GGC GGG GCG GGG GCA CGG GGG GCC TAC GGG AGG CAG CAG-3'; 
R518, 5'-ATT ACC GCG GCT GCT GG-3'). A GC-clamp (CGC CCG CCG CGC GCG GGC GGG GCG GGG GCA CGG GGG GCC) was applied to the 5'-end of the forward primer to increase the sensitivity of DGGE analysis. PCR amplification was performed using a hot start and touchdown PCR. The following conditions were used: hot start of $3 \mathrm{~min}$ at $94^{\circ} \mathrm{C} ; 20$ cycles consisting of $94^{\circ} \mathrm{C}$ for $30 \mathrm{~s}, 65^{\circ} \mathrm{C}$ for $30 \mathrm{~s}$, decreasing the temperature by $0.5^{\circ} \mathrm{C}$ per cycle (touchdown $\mathrm{PCR}$ ), $72^{\circ} \mathrm{C}$ for $30 \mathrm{~s} ; 15$ cycles consisting of $94^{\circ} \mathrm{C}$ for $30 \mathrm{~s}, 53^{\circ} \mathrm{C}$ for $30 \mathrm{~s}, 72^{\circ} \mathrm{C}$ for $30 \mathrm{~s}$; a final step of $7 \mathrm{~min}$ at $72^{\circ} \mathrm{C}$. To construct library of $16 \mathrm{~S}$ rRNA genes, nearly full-length 16S rRNA products were amplified using the $P 1$ and $P 2$ universal primers of $16 \mathrm{~S}$ rRNA genes (P1, 5'-AGA GTT TGA TCC TGG CTC AGA ACG AAC GCT-3'; P2, 5'-TAC GGC TAC CTT GTT ACG ACT TCA CCC C-3') (Brosius et al., 1978). The reaction program was as follows: $3 \mathrm{~min}$ at $95^{\circ} \mathrm{C}$, followed by 25 cycles for $30 \mathrm{~s} \mathrm{~min}$ at $95^{\circ} \mathrm{C}, 30 \mathrm{~s}$ at $60^{\circ} \mathrm{C}$, and $2 \mathrm{~min}$ at $72^{\circ} \mathrm{C}$, with a final extension for $10 \mathrm{~min}$ at $72^{\circ} \mathrm{C}$. All amplification products were separated by $1 \%$ agarose gel electrophoresis and stained with ethidium bromide. The DNA bands of approximately $180 \mathrm{bp}$ for the $\mathrm{V} 3$ region and $1500 \mathrm{bp}$ for $16 \mathrm{~S}$ were excised and purified using the SanPrep Gel Extraction Mini Kit (Sangon Biotech, Shanghai, China).

\section{DGGE}

The PCR products of the V3 region for the 16S rRNA genes were electrophoretically separated by DGGE using the Bio-Rad Dcode mutation detection system (Hercules, CA, USA) according to the manufacturer instructions. Next, $40 \mu \mathrm{L}$ of each PCR product was loaded on $10 \%$ $(\mathrm{w} / \mathrm{v})$ polyacrylamide gels with a denaturing gradient from $30-60 \%$ and containing $12.6-25.2 \%(\mathrm{w} / \mathrm{v})$ urea and $12-24 \%(v / v)$ deionized formamide (Sangon Biotech). Electrophoresis was performed in $1 \mathrm{X}$ TAE buffer for $10 \mathrm{~min}$ at a constant voltage $200 \mathrm{~V}$ at $60^{\circ} \mathrm{C}$, and then at $85 \mathrm{~V}$ for $16 \mathrm{~h}$. The gel was stained with $0.5 \mu \mathrm{L} / \mathrm{mL}$ ethidium bromide for $20 \mathrm{~min}$, rinsed with deionized water for 20 min, and photographed. Characteristic bands were removed from the gel. The small gel pieces were rinsed with $100 \mu \mathrm{L} 70 \%$ precooled ethanol 3-4 times, and then $50 \mu \mathrm{L}$ double-distilled $\mathrm{H}_{2} \mathrm{O}$ was added to the gel pieces after they were air-dried and soaked for approximately $10 \mathrm{~h}$ at $4^{\circ} \mathrm{C}$. PCR was conducted for the recovered DNA fragments under the same conditions as were used for the V3 region, and detected and purified by DGGE. Next, the recovered DNA fragments were amplified in the V3 region-specific primers F341 (5'-ACG GGG GGC CTA CGG GAG GCA G-3') and R518 (5'-ATT ACC GCG GCT GCT GG-3') without a GC-clamp. The PCR products were sequenced by Sangon Biotech.

\section{Clone library construction}

Purified PCR products of nearly full-length $16 \mathrm{~S}$ rRNA were ligated into the PMD $^{\mathrm{TM}} 19-\mathrm{T}$ vector according to the manufacturer instructions (TaKaRa, Shiga, Japan) and transformed into Escherichia coli $\mathrm{DH} 5 \alpha$ cells. A total of $100-\mu \mathrm{L}$ transformation reaction was plated on an LB plate containing ampicillin, isopropyl $\beta$-D-1-thiogalactopyranoside, and X-Gal and the plates were incubated overnight at $37^{\circ} \mathrm{C}$. White clones were picked and amplified using the universal primer P1/P2 according to the method described by Zoetendal et al. (1998). Inserts of the correct 
size were considered to have come from positive clones. Several positive clones were selected randomly and amplified using the primers F341-GC and R518, and grouped into operational taxonomic units (OTUs) by DGGE. For OTUs with more than 30 clones, 5 representative clones were randomly selected and sequenced by Sangon Biotech. For OTUs with fewer than 6 clones, all clones were sent for sequencing. For OTUs with more than 6 clones, 3 or 4 clones were randomly selected for sequencing.

\section{Contrastive analysis of images and sequences}

All DGGE images were exported as uncompressed TIF files and analyzed using the Quantity One software (Bio-Rad). Sorenson's similarity index and unweighted pair group method with arithmetic mean were determined by the Quantity One software, while the Shannon-Wiener index, Jaccard index, and Cody index were determined from the data exported from Quantity One. In addition, we determined the relative $Q$-value of each band in each lane and obtained the relative abundance. Additionally, sequencing results were compared with those available in GenBank to identify the closest relatives using the BLAST algorithm. Three phylogenetic trees were constructed using the neighbor-joining method with the TREECONW software packages. The partial 16S rRNA gene sequences from the clone library were deposited in the GenBank database and assigned accession numbers KF145967-KF145981.

\section{RESULTS}

\section{DGGE profiles and their sequencing}

In order to understand the gut microbial community composition in M. shangchengensis and 3 groups of $T$. ampliceps, we conducted DGGE analysis of the $\mathrm{V} 3$ regions of $16 \mathrm{~S}$ rRNA amplified from total DNA extracted from gut samples (Figure 1). Approximately 20 DGGE bands were observed on the DGGE profile of $T$. ampliceps (Figure $1 \mathrm{~A}$ ) and $M$. shangchengensis (Figure 1B). Each sample profile displayed a unique banding pattern. The results showed that the gut microbial communities' diversity and relative abundance in T. ampliceps were influenced by the diet materials containing different components. Furthermore, an unweighted pair group method with arithmetic mean dendrogram (Figure 1C) determined by cluster analysis using the Quantity One software revealed that the 2 groups fed with corn stalk and filter paper were very similar to each other, and 1 clade associated with the 2 groups with a coefficient of 0.73 , which is more than 0.53 .

Eighteen and 16 sequences obtained from the samples were compared with other sequences available in the NCBI database; relative abundance was analyzed using the Quantity One software (Tables 1 and 2) to identify differences between samples from the 2 types of termites and changes caused by feeding of different substrates. Sequence analysis showed that the intestine of termites was dominated by Proteobacterium, Firmicutes, Actinobacteria, Fibrobacteres, Spirochaetes, Bacteroidetes, Deferribacteres, and some protozoans or protozoan symbionts. Protozoan symbionts were only observed in the samples from lower termites. 
A

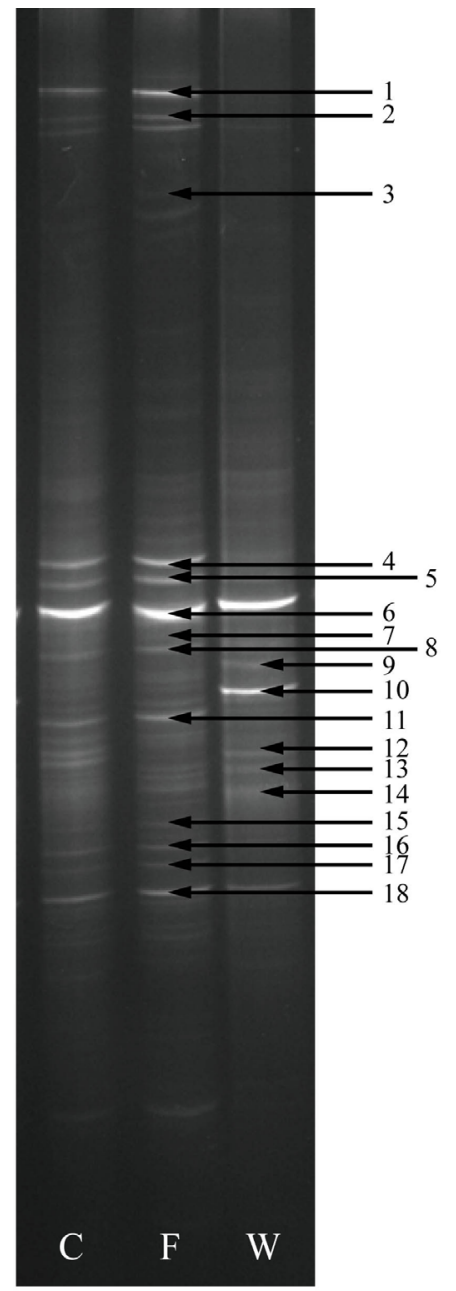

B

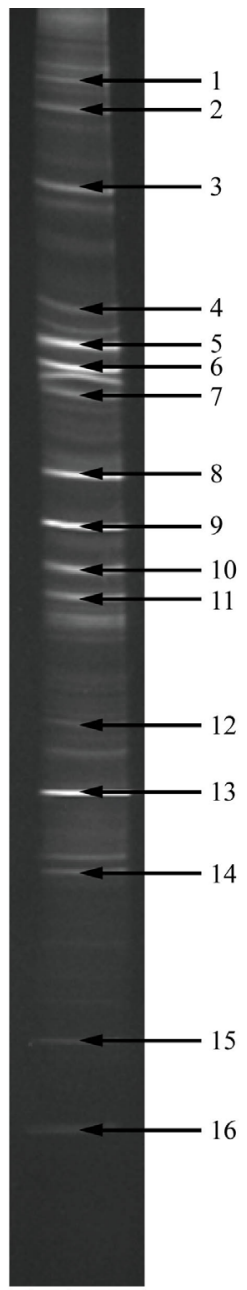

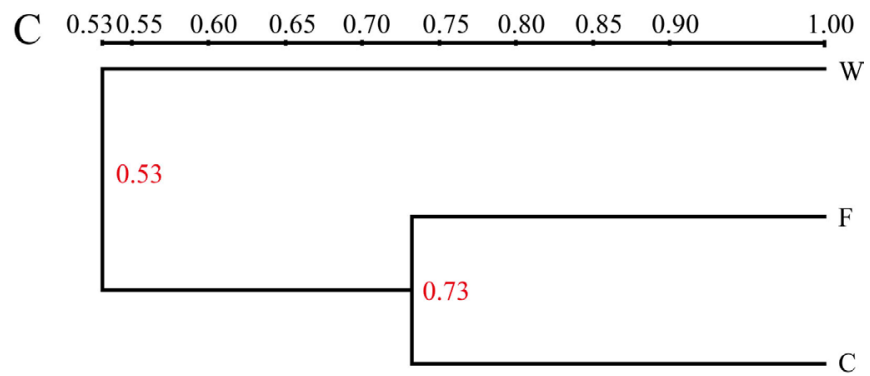

Figure 1. Denaturing gradient gel electrophoresis (DGGE) profiles. A. DGGE profiles of the $\mathrm{V} 3$ region amplified by PCR from DNA extracted from the gut samples of Tsaitermes ampliceps fed with different materials (C: corn stalk; F: filter paper; W: Robinia pseudoacacia); B. DGGE profiles of the V3 region amplified by PCR from the DNA extracted from the gut samples of Mironasutitermes shangchengensis; C. Hierarchical cluster analysis results of Figure $1 \mathrm{~A}$ demonstrated graphically as an unweighted pair group with arithmetic mean dendrogram. 
Table 1. Homologous sequence, identity, accession number in GenBank, and relative abundance of the major bands in Tsaitermes ampliceps from the 16S rRNA V3 DGGE shown as in Figure 1A.

\begin{tabular}{|c|c|c|c|c|c|}
\hline \multirow[t]{2}{*}{ Band No. } & \multirow[t]{2}{*}{ Homologous sequence in GenBank } & \multirow[t]{2}{*}{ Identity (\%) } & \multicolumn{3}{|c|}{ Relative abundance (\%) } \\
\hline & & & W & $\mathrm{C}$ & $\mathrm{F}$ \\
\hline & Bacteroidetes & & 0 & 20.63 & 23.03 \\
\hline T.a 1 & Uncultured Bacteroidetes bacterium clone Cf2-21(GQ502490.1) & 93 & $0^{\mathrm{A}}$ & $10.51^{\mathrm{B}}$ & $8.79^{\mathrm{B}}$ \\
\hline T.a 2 & Uncultured Bacteroidetes bacterium clone: RsStar413(AB522116.1) & 93 & $0^{A}$ & $5.45^{\mathrm{B}}$ & $4.24^{\mathrm{B}}$ \\
\hline T.a 3 & Uncultured Bacteroidales bacterium clone: RsTu1-23(AB192206.1) & 95 & $0^{\mathrm{A}}$ & $0^{A}$ & $4.85^{\mathrm{B}}$ \\
\hline \multirow[t]{2}{*}{ T.a 15} & Uncultured Bacteroidetes bacterium clone CTF2-117(GU959229.1) & 91 & $0^{\mathrm{A}}$ & $4.67^{\mathrm{B}}$ & $5.15^{\mathrm{B}}$ \\
\hline & Proteobacterium & & 52.54 & 35.8 & 44.55 \\
\hline T.a 4 & Uncultured epsilon proteobacterium clone Rc238 (JQ617848.1) & 94 & $0^{\mathrm{A}}$ & $11.28^{\mathrm{B}}$ & $8.48^{\mathrm{B}}$ \\
\hline T.a 5 & Uncultured Sulfurimonas sp clone BFB083 (KC545729.1) & 96 & $10.17^{\mathrm{a}}$ & $10.51^{\mathrm{a}}$ & $7.88^{\mathrm{b}}$ \\
\hline T.a 7 & Uncultured Escherichia sp clone 7d14221 (GU132148.2) & 95 & $0^{\mathrm{a}}$ & $0^{\mathrm{a}}$ & $5.76^{\mathrm{B}}$ \\
\hline T.a 8 & Uncultured Escherichia sp (AM712054.1) & 98 & $10.17^{\mathrm{A}}$ & $14.01^{\mathrm{b}}$ & $9.70^{\mathrm{a}}$ \\
\hline T.a 9 & Uncultured alpha proteobacterium clone Rc282 (JQ617836.1) & 95 & $11.44^{\mathrm{A}}$ & $0^{\mathrm{B}}$ & $9.09^{\mathrm{a}}$ \\
\hline \multirow[t]{2}{*}{ T.a 10} & Uncultured Escherichia sp (AM748793.1) & 100 & $20.76^{\mathrm{A}}$ & $0^{\mathrm{B}}$ & $3.64^{\mathrm{C}}$ \\
\hline & Protozoa & & & & \\
\hline \multirow[t]{2}{*}{ T.a 11} & Amblyospora sp (U68474.1) & 88 & $8.47^{\mathrm{a}}$ & $8.56^{\mathrm{a}}$ & $6.97^{\mathrm{a}}$ \\
\hline & Firmicutes & & 20.76 & 12.06 & 7.27 \\
\hline T.a 12 & Uncultured Eubacteriaceae bacterium clone: Rs-N82 (AB088960.2) & 93 & $8.05^{\mathrm{A}}$ & $4.28^{\mathrm{B}}$ & $3.03^{\mathrm{B}}$ \\
\hline \multirow[t]{2}{*}{ T.a 14} & Bacillus sp C271 (AB741803.1) & 80 & $12.71^{\mathrm{A}}$ & $7.78^{\mathrm{b}}$ & $4.24^{\mathrm{C}}$ \\
\hline & Deferribacteres & & & & \\
\hline \multirow[t]{2}{*}{ T.a 13} & Uncultured Deferribacteraceae bacterium clone SL70 (JN680689.1) & 88 & $9.32^{\mathrm{A}}$ & $0^{\mathrm{B}}$ & $5.45^{\mathrm{A}}$ \\
\hline & Spirochaetes & & 8.91 & 22.96 & 12.73 \\
\hline T.a 16 & Uncultured Spirochaetes bacterium clone Rc342 (JQ617773.1) & 100 & $0^{\mathrm{A}}$ & $5.06^{\mathrm{B}}$ & $5.15^{\mathrm{B}}$ \\
\hline T.a 17 & Uncultured Treponema sp clone 290cost002-P3L-1298 (EF453910.2) & 97 & $0^{\mathrm{a}}$ & $3.89^{\mathrm{a}}$ & $0^{\mathrm{a}}$ \\
\hline T.a 18 & Uncultured Treponema sp clone rG16 (HQ187677.1) & 96 & $8.91^{\mathrm{a}}$ & $14.01^{\mathrm{B}}$ & $7.58^{\mathrm{a}}$ \\
\hline T.a $6^{*}$ & Incisitermes snyderi (GQ337717.1) & 97 & $20^{\mathrm{a}}$ & $24.41^{b}$ & $13.61^{\mathrm{C}}$ \\
\hline
\end{tabular}

*The sequence belonged to $T$. ampliceps, so the relative abundance of each operational taxonomic unit was calculated without the data of T.a 6 . The letters marked on the data indicate significance levels, where lowercase letters represent significance with $\mathrm{P}<0.05$ and uppercase letters represent very significant values with $\mathrm{P}<0.01$. W $=$ Robinia pseudoacacia; $\mathrm{C}=$ corn stalk; $\mathrm{F}=$ filter paper.

Table 2. Homologous sequence, identity, accession number in GenBank, and relative abundance for the major bands in Mironasutitermes shangchengensis from the 16S rRNA V3 DGGE shown as in Figure 1B.

\begin{tabular}{|c|c|c|c|}
\hline Band No. & Homologous sequence in GenBank & Identity (\%) & Relative abundance (\%) \\
\hline & Proteobacterium & & 51.73 \\
\hline M.s 1 & Enterobacter sp SBS1 (KC758848.1) & 98 & 5.2 \\
\hline M.s 2 & Klebsiella oxytoca strain A11n (KC513855.1) & 98 & 4.46 \\
\hline M.s 4 & Enterobacter sp NCCP-242 (AB665216.1) & 98 & 4.21 \\
\hline M.s 5 & Enterobacter sp Hb-0512 (GQ487560.1) & 99 & 7.9 \\
\hline M.s 6 & Enterobacter sp clone CD01013C12 (HM122394.1) & 99 & 9.41 \\
\hline M.s 7 & Comamonas sp UVS1 (AM503546.1) & 98 & 6.44 \\
\hline M.s 8 & Uncultured delta proteobacterium clone RsStar232 (AB522152.1) & 95 & 8.91 \\
\hline \multirow[t]{2}{*}{ M.s 14} & Uncultured beta proteobacterium clone RsW02-065 (AB198538.1) & 91 & 5.2 \\
\hline & Firmicutes & & 13.86 \\
\hline M.s 3 & Anoxybacillus flavithermus strain CM (KC508690.1) & 91 & 4.7 \\
\hline M.s 11 & Uncultured Lachnospiraceae bacterium clone FS_46.41-1 (GU939256.1) & 85 & 5.69 \\
\hline \multirow[t]{2}{*}{ M.s 15} & Uncultured Lactococcus sp clone RsStar323 (AB522148.1) & 86 & 3.47 \\
\hline & Actinobacteria & & \\
\hline \multirow[t]{2}{*}{ M.s 9} & Uncultured Actinomycete clone MgMjR-011 (AB234517.1) & 99 & 8.42 \\
\hline & Fibrobacteres & & 14.11 \\
\hline M.s 10 & Uncultured Fibrobacteres bacterium Nd-Fib-2 (AB255951.1) & 95 & 7.43 \\
\hline \multirow[t]{2}{*}{ M.s 12} & Uncultured Fibrobacteres clone Nt2-023 (AB255932.1) & 93 & 6.68 \\
\hline & Spirochaetes & & \\
\hline \multirow[t]{2}{*}{ M.s 13} & Uncultured Treponema sp clone 290cost002-P3L-1298 (EF453910.2) & 96 & 8.17 \\
\hline & Bacteroidetes & & \\
\hline M.s 16 & Uncultured Bacteroidales clone RsW01-089 (AB198512.1) & 94 & 3.71 \\
\hline
\end{tabular}




\section{S rRNA gene clone library}

A nearly full-length of $16 \mathrm{~S}$ rRNA gene clone library was constructed from $T$. ampliceps guts consisting of 507 positive clones, which were randomly selected and tested by colony PCR. The positive rate was approximately $74.6 \%$. All 378 positive clones were separated into 15 different hyplotypes by DGGE, and almost the full-length of the16S rRNA gene was sequenced and analyzed (Table 3). All sequences showed sequence identities of more than $92 \%$ with sequences that had been submitted to NCBI and GenBank accession numbers were assigned (Table 3). The sequences revealed a large number of microbial communities and that the clones of the sequences mainly belonged to Spirochaetes (28.07\%), Firmicutes (26.46\%), Proteobacteria (22.22\%), and Protozoan symbionts (23.28\%).

\begin{tabular}{|c|c|c|c|c|}
\hline Accession No. & Homologous sequence in Genbank & Identity (\%) & $\begin{array}{l}\text { Numbers } \\
\text { of Clones }\end{array}$ & $\begin{array}{c}\text { Relative } \\
\text { abundance (\%) }\end{array}$ \\
\hline & Spirochaetes & & & 28.04 \\
\hline KF145967 & Uncultured Spirochaetes bacterium clone Rc342 (JQ617773.1) JQ617773.1 & 99 & 34 & 8.99 \\
\hline KF145972 & Uncultured Spirochaeta sp gene clone: Rs-H09 (AB088910.1) & 99 & 32 & 8.47 \\
\hline KF145977 & Uncultured Spirochaetes bacterium clone Rc12 (JQ617747.1) & 99 & 28 & 7.41 \\
\hline \multirow[t]{2}{*}{ KF145981 } & Uncultured Treponema sp gene clone: Rs-P25 (AB088882.2) & 97 & 12 & 3.17 \\
\hline & Proteobacteria & & & 22.22 \\
\hline KF145968 & Uncultured alpha Proteobacterium gene clone: RsStar117 (AB522149.1) & 97 & 20 & 5.29 \\
\hline KF145969 & Uncultured alpha Proteobacterium gene clone: RPK-49 (AB192282.1) & 98 & 22 & 5.82 \\
\hline KF145973 & Uncultured delta Proteobacterium gene clone: HsTph-09 (AB444077.1) & 99 & 16 & 4.23 \\
\hline \multirow[t]{2}{*}{ KF145978 } & Uncultured delta Proteobacterium clone Rc328 (JQ617846.1) & 95 & 26 & 6.88 \\
\hline & Firmicutes & & & 26.46 \\
\hline KF145970 & Uncultured Clostridiales bacterium clone: MFeClo012 (AB702886.1) & 90 & 34 & 8.99 \\
\hline KF145974 & Uncultured Clostridiales bacterium clone: RsW01-035 (AB198470.1) & 99 & 21 & 5.56 \\
\hline KF145975 & Uncultured Eubacteriaceae bacterium clone: RPK-55 (AB192278.1) & 99 & 16 & 4.23 \\
\hline KF145979 & Uncultured Clostridiaceae bacterium clone MTG-3 (DQ307710.1) & 92 & 21 & 5.56 \\
\hline \multirow[t]{2}{*}{ KF145976 } & Lactococcus sp TSB11 (JX291542.1) & 98 & 8 & 2.12 \\
\hline & Endosymbiont of Protozoa & & & 23.28 \\
\hline KF145971 & Uncultured endosymbiont of Pyrsonympha vertens clone RfPv16 (AY572024.1) & 95 & 46 & 12.17 \\
\hline KF145980 & Uncultured endosymbiont of Pyrsonympha vertens clone RfPv15(AY572023.1) & 95 & 42 & 11.11 \\
\hline
\end{tabular}

\section{Linking dietary patterns with gut microbial of termites}

The food effect was examined by comparing the DGGE lanes from the 3 diet groups (Figure 1A). Table 1 shows that large changes occurred in the relative abundance of the 3 groups (W, C, and $F$ ), and statistical analyses showed that 2-week diet shifts significantly impacted microbiota composition. The results of Student $t$-tests in Table 1 showed that most of the differences in species between the gut microflora of termite feeding with wood diet and feeding with corn stalk diet reached very significant levels $(P<0.01)$, particularly within Bacteroidetes, Spirochaetes, and Firmicutes. In addition, we also calculated the diversity indices including Shannon-Wiener index and $3 \beta$-diversity indices, including Sorenson's similarity index, Jaccard index, and Cody index (Table 4). Although the rate of community species change was low $(C j \leq 4.5)$, the similarity indices between 2 of the 3 groups were also low (51.5-61.8, 43.8-66.7). Moreover, the Shannon-Wiener index increased progressively $(2.15,2.40$, and 2.72$)$ with the 3 groups. Wood-fed workers showed $40 \%$ lower community diversity than cellulose-fed workers of Centrocestus formosanus fed for 30 days (Tanaka et al., 2006), while our results suggest that this value is $43.75 \%$. Additionally, in the case of worker termites feeding on cellulose, the number of some protozoa decreased when compared to those feeding on wood and wood powder. According to Table 1, the protozoan 
symbionts also reduced. The results of the indexes $(P<0.01)$ suggested consistently differences in gut bacterial community composition as a result of diet.

The sequence of T.a 6 (Table 1) belonged to T. ampliceps. As shown in Figure 2, we determined that the surface of the peaks of T.a 6 (Table 1) for the 3 groups were approximately the same size. This indicates that the total number of microorganisms was the lowest in the gut of group fed with corn stalk with T.a 6 at $24.41 \%$, while group fed with filter paper showed the highest numbers of microorganism with band 6 at $13.61 \%$.

\begin{tabular}{|c|c|c|c|c|c|c|c|c|c|c|c|c|}
\hline & & \multirow{2}{*}{$\begin{array}{l}\text { Numbers } \\
\text { of phylotypes }\end{array}$} & \multirow{2}{*}{$\begin{array}{c}\text { Shannon- } \\
\text { Wiener index }\end{array}$} & \multicolumn{3}{|c|}{ Sorenson's } & \multicolumn{3}{|c|}{ Jaccard index (\%) } & \multicolumn{3}{|c|}{ Cody index } \\
\hline & & & & W & C & $\mathrm{F}$ & W & $\mathrm{C}$ & $\mathrm{F}$ & W & $\mathrm{C}$ & $\mathrm{F}$ \\
\hline \multirow[t]{3}{*}{ T. ampliceps } & W & 9 & 2.15 & 100.0 & & & 100.0 & & & 0.0 & & \\
\hline & C & 12 & 2.40 & 61.8 & 100.0 & & 43.8 & 100.0 & & 4.5 & 0 & \\
\hline & $\mathrm{F}$ & 16 & 2.72 & 51.5 & 61.1 & 100.0 & 58.8 & 66.7 & 100.0 & 3.5 & 3 & 0 \\
\hline
\end{tabular}

These indexes were calculated using the following equation: Shannon - Wiener index $\left(H^{\prime}\right)=-\sum_{i}^{s}(P i)(\ln P i)$ where $s$ is the number of species in the sample and $P i$ is the proportion of species $i$ in the sample. Pi could be calculated by the relative surface of the peak in the DGGE profile $(\mathrm{Pi}=\mathrm{Ni} / \mathrm{N}$, where $\mathrm{Ni}$ is the surface of the peak, and $\mathrm{N}$ is the sum of the surfaces for all peaks within the lane), Sorenson's similarity index $(S I)=2($ nal $n b) / n a+n b$; Jaccard index $(C j)=(n a l n b) / n a+n b ;$ Cody index $(\beta c)=(\mathrm{na}+\mathrm{n} b-2 \mathrm{nal} \mathrm{nb}) / 2$, where na and $\mathrm{n} b$ are numbers of bands in different lanes. $\mathrm{W}=$ Robinia $p s e u d o a c a c i a ;$ $\mathrm{C}=$ corn stalk; $\mathrm{F}=$ filter paper.

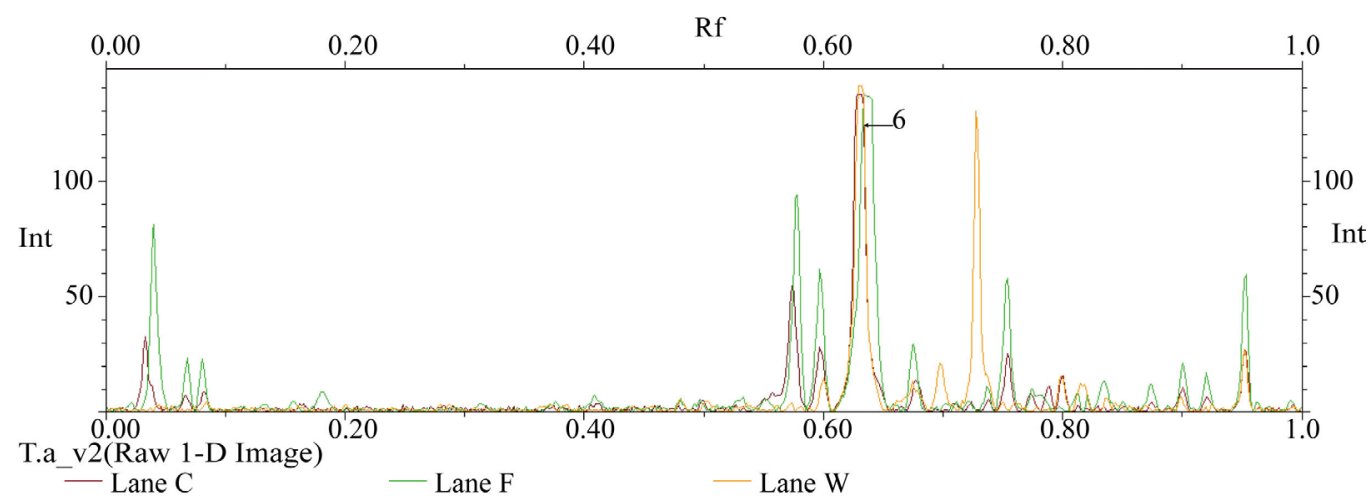

Figure 2. Lanes compared using the Quantity One software (Bio-Rad) from Figure 1A. Lane $C$ with the red line represents the group fed with corn stalk; lane $F$ with the green line represents the group fed with filter paper, and lane $W$ is the group fed with Robinia pseudoacacia.

\section{Comparing diversity of termite gut microbiota}

Table 5 shows the phylotypes within the 3 major phyla (Spirochaetes, Firmicutes, and Proteobacteria) and others detected in the termite gut. The number and relative abundance of phylotypes within Proteobacteria was the largest among the 6 phyla in M. shangchengensis, while the number of phylotypes within Firmicutes and Proteobacteria in T. ampliceps were higher than in others. Higher termites not only shared the same 3 major phyla with $T$. ampliceps, but also showed the same phylotypes within Bacteroidetes, Actinobacteria, and Fibrobacteres. However, the phylum of protozoan symbionts was observed in large numbers in lower termites. In addition, the Shannon- 
Wiener index showed the highest diversity of gut microbial communities in $M$. shangchengensis, with an index value of 2.72. These data indicate remarkably abundant protozoan symbionts in the gut of lower termites, and higher termites showed more complex and diversity for the intestinal symbiont environment. The phylotypes of the sequences showed only a few differences between the 2 sets of $T$. ampliceps, but the number and the relative abundance of the phylotypes and the diversity index were not similar. The number of phylotypes in the clone library was much larger than that of sequences determined by DGGE; the relative abundance of Spirochaetes and Proteobacteria of the 3 major phyla showed large changes. This may be attributable because of the method used for testing: both methods inevitably lost a few of phylotypes; however, DGGE had the advantages of reflecting diversity of the intestinal flora, while 16S rRNA library analyses were used to obtain full-length sequences of the 16S rRNA gene to identify the species present.

Table 5. Phylotypes and relative abundance of gut microbe in termite colonies.

\begin{tabular}{|c|c|c|c|c|c|c|}
\hline & \multicolumn{2}{|c|}{ M. shangchengensis } & \multicolumn{2}{|c|}{ T. ampliceps } & \multicolumn{2}{|c|}{ T. ampliceps } \\
\hline & $\begin{array}{c}\text { No. of } \\
\text { phylotypes }\end{array}$ & $\begin{array}{c}\text { Relative } \\
\text { abundance (\%) }\end{array}$ & $\begin{array}{c}\text { No. of } \\
\text { phylotypes }\end{array}$ & $\begin{array}{c}\text { Relative } \\
\text { abundance (\%) }\end{array}$ & $\begin{array}{c}\text { No. of } \\
\text { phylotypes }\end{array}$ & $\begin{array}{c}\text { Relative } \\
\text { abundance (\%) }\end{array}$ \\
\hline Firmicutes & 3 & 13.86 & 2 & 20.76 & 5 & 26.46 \\
\hline Proteobacteria & 8 & 51.73 & 4 & 52.54 & 4 & 22.22 \\
\hline Spirochaetes & 1 & 8.17 & 1 & 8.90 & 4 & 28.04 \\
\hline Bacteroidetes & 1 & 3.71 & & & & \\
\hline Actinobacteria & 1 & 8.42 & & & & \\
\hline Fibrobacteres & 2 & 14.11 & & & & \\
\hline Deferribacteres & & & 1 & 9.32 & & \\
\hline Protozoa & & & 1 & 8.48 & 2 & 23.28 \\
\hline Total & $6 / 6 / 16$ & 100.00 & $4 / 5 / 11$ & 100.00 & $3 / 4 / 15$ & 100.00 \\
\hline Shannon-Wiener index & 2.72 & & 2.15 & & 2.62 & \\
\hline
\end{tabular}

${ }^{*} T$. ampliceps: Tsaitermes ampliceps; M. shangchengensis: Mironasutitermes shangchengensis.

\section{DISCUSSION}

In this study, we found that higher termites $M$. shangchengensis had a more complex and diversity intestinal environment than did lower termites $T$. ampliceps. The 2-week diet shifts significantly impacted microbiota composition, including both the number and the relative abundance of the phylotypes.

Analyzed sequences were used to construct a phylogenetic tree (Figure 3). Most sequences were very close to each other in the tree and were gathered into groups, which respectively represented Spirochaetes, Bacteroidetes, Proteobacteria, and others. Members of 7 bacterial phyla were detected in the microhabitat of the 2 types of termite hindgut lumen, with more than $76 \%$ belonging to Spirochaetes, Firmicutes, Proteobacteria, Bacteroidetes, Actinobacteria, Fibrobacteres, and Deferribacteres. For some protozoa found in $T$. ampliceps, the sequence similarity of results obtained by DGGE and the clone library were lower, which may have resulted from limitations of the method itself.

Previous studies identified Spirochaetes as a major component of the lower termite hindgut microbiome (Hongoh, 2003; Warnecke et al., 2007; Husseneder et al., 2010). They exist in the hindgut and are recognized as host-specific ectosymbionts of various protozoans inhabiting the termite hindgut (lida et al., 2000; Ohkuma, 2008). These organisms play an important role in encoding for an array of hydrolytic enzymes involved in cellulose digestion (Warnecke et al., 2007) and have $\mathrm{CO}_{2}$-reducing acetogenesis activity (Leadbetter and Breznak, 1996). Studies also have 
shown that select ectosymbiotic spirochetes associated with the protist Mixotricha paradoxa provide motility (Cleveland and Grimstone, 1964). This conclusion has been used as a strong evidence of the endosymbiosis hypothesis. In this study, we not only found both Treponema-related and Spirochetes-related phylotypes, but also obtained a sequence marked as T.a 17, which showed $90 \%$ sequence similarity to the existing rod-shaped symbiont of Mixotricha paradoxa (AJ488195.1) in GenBank. This organism was present in both T. ampliceps and M. shangchengensis, and increased to $22.96 \%$ in $T$. ampliceps after the diet change. Thus, Spirochaetes play an important role in degrading lignocellulose, particularly cellulose.

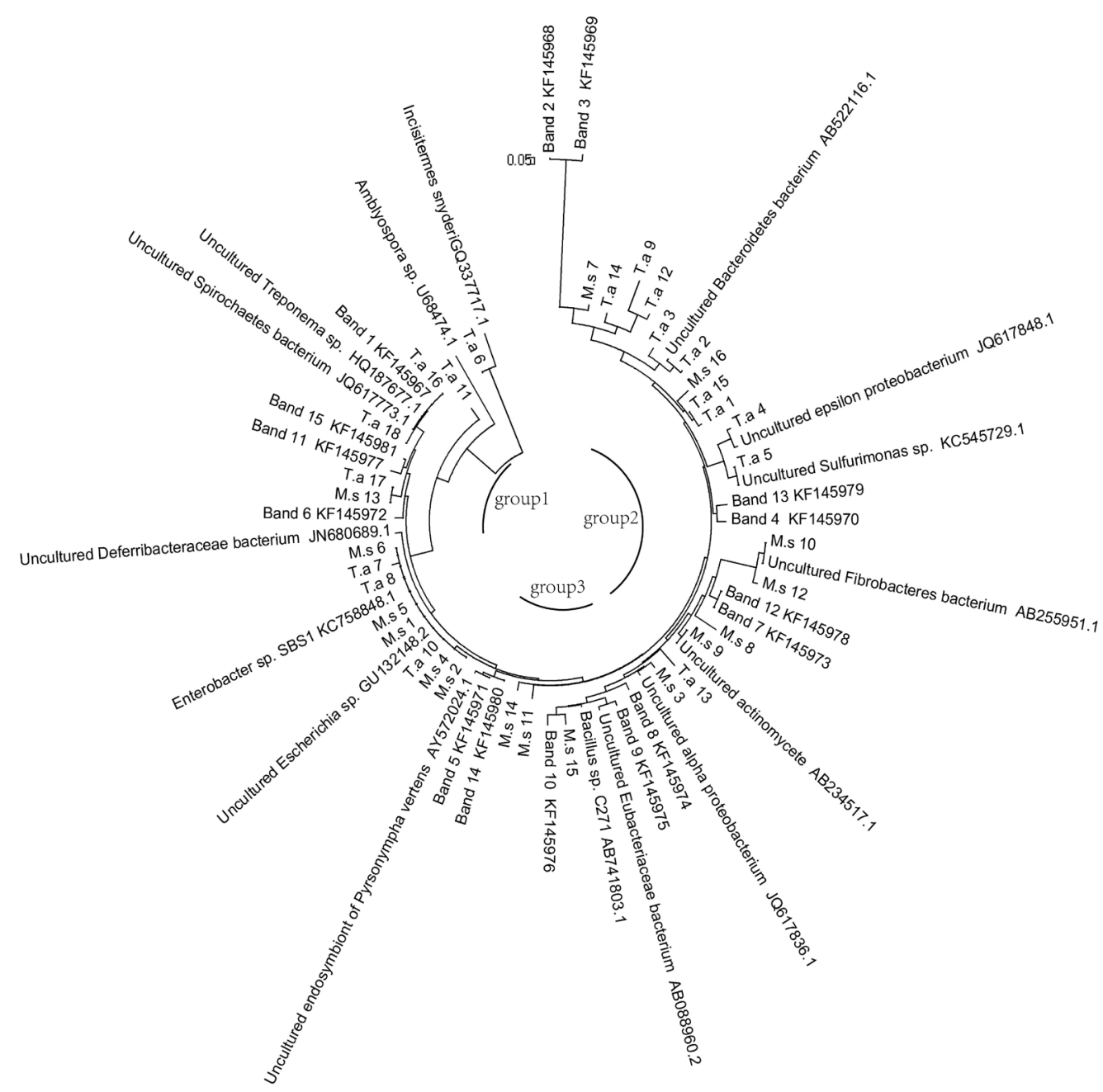

Figure 3. Dendrogram of $16 \mathrm{~S}$ rRNA gene or V3 region of $16 \mathrm{~S}$ rRNA gene sequences showing the phylogenetic affiliation of intestinal bacteria in Tsaitermes ampliceps and Mironasutitermes shangchengensis. A neighbor-joining tree was constructed from all gene sequences obtained in this study of each phylotype and from sequences retrieved from the GenBank database. T.a represents the sequence acquired from T. ampliceps by DGGE, while M.s represents the sequence from M. shangchengensis. Groups 1, 2, and 3 represent Spirochaetes, Bacteroidetes, and Proteobacteria, respectively. Other details are the same as in the tables above. 
The phylum Firmicutes contained members that could be annotated to the orders Clostridiales and Bacillales. Several phylotypes within the Clostridiales group were annotated to the Lachnospiraceae, Clostridiales, and Eubacteriaceae species, known for their ability to digest cellulose. Clostridiales have been reported to be affiliated with the intestinal lining of the hindgut (Yang et al., 2005), and thus may participate in digestion on the intestinal lining of the termite hindgut. Members of Bacillales belonged to Lactococcus, Bacillus, and Anoxybacillus flavithermus. Lactococcus produces $\alpha$-galactosidases and likely plays a role in digesting both galactosecontaining oligosaccharides and polymeric galacto(gluco)mannans in hemicelluloses (Boucher et al., 2003). A. flavithermus was able to utilize a wide range of carbon sources, such as glucose, xylose, arabinose, starch, maltose, and sorbitol, and anaerobically fermented ethanol as a minor product (Peng et al., 2008).

Bacteroidetes are both ecto- and endosymbionts of hindgut protozoans in lower termites (Noda et al., 2006). However, we discovered Bacteroidetes in higher termites of M. shangchengensis. Bacteroidetes may function without hindgut protozoans in higher termites. Moreover, in the lower termites of $T$. ampliceps fed with filter paper and corn stalk, more Bacteroidetes were observed $(>23 \%)$ than in termites fed on wood. Thus, celluloses rather than lignin was the substrate on which Bacteroidetes acted.

In this study, the Fibrobacteres was separated phylogenetically from only $M$. shangchengensis. This poorly defined phylum includes species involved in cellulose digestion in ruminant animals (Brumm et al., 2011). Fibrobacteres associated with $R$. flavipes represent novel members affiliated with subphylum 2 (Hongoh et al., 2005, 2006). Warnecke et al. (2007) and Engelbrektson et al. (2010) supported these findings by showing that Fibrobacteres was the second-most abundant bacterial group in Nasutitermes.

Proteobacteria contained members annotated to the $\alpha, \beta, \gamma, \delta$, and $\varepsilon$ sections, which were ubiquitous inhabitants of insect digestive tracts with the relative abundances of 52.54, 35.8, 44.55, 22.22 , and $51.73 \%$. Jiao et al. (2006) revealed a Proteobacteria rhodopsin, which converted light energy into a chemical potential gradient of protons inside and outside the cell membrane for the synthesis of ATP. Eight groups of Proteobacteria with nitrogenase activity were isolated from Oryza rufipogon (Tan et al., 2009). Furthermore, the bacterial nitrification gene (hao) was obtained from the $\alpha, \beta, \gamma$-Proteobacteria. Thus, Proteobacteria has a wide range of functions in photosynthesis, bacteriolysis, nitrification, and azotification, which may be significant for balancing energy and the milieu internee in the gut of termites.

Previous studies found that a large number of symbiotic protozoa, such as flagellates, settled in the posterior intestine in some lower termites. A study on Reticulitermes chinensis Snyder found $70 \%$ more symbiotic Oxymonad flagellates (Chen et al., 2011). An American study listed the intestinal symbiotic types of giardia in 205 kinds of wood-feeding lower termites and found 430 types of symbiotic protozoa that play an important role in fiber depolymerization and fermentation. We also found a high abundance of protozoan symbionts in the intestinal tract of $T$. ampliceps, indicating that protozoa plays important roles in the decomposition, digestion, and metabolism of diets in T. ampliceps.

By comparing microbiota composition after the 2-week feeding of diets containing different lignocellulose compositions, we found that changes in food composition affected the relative abundance and diversity of enteric microorganisms. When fed with wood with a higher content of lignin, Bacteroidetes and Spirochaetes showed the lowest number and relative abundance, indicating that these phyla consume celluloses rather than lignin; Deferribacteres, Firmicutes, and Protozoa were most abundant in the group fed with wood, suggesting that they mainly act on lignin 
rather than celluloses. Proteobacteria retained the dominant position because of its wide range of functions in energy production and gut environment balance.

Furthermore, Spirochaetes and protozoa were the main groups that digest lignocellulose in $T$. ampliceps, and they decompose and ferment cellulose into acetic acid, $\mathrm{CO}_{2}$, and $\mathrm{H}_{2}$; next, the Treponema-related and Spirochetes-related phylotypes deoxidate $\mathrm{CO}_{2}$ into acetic acid. Hence, termites are provided with the nutrition and energy to survive (Warnecke et al., 2007; Brune, 2014). Proteobacteria also play vital function in supporting termite survival and metabolism. There were nearly no protozoa in $M$. shangchengensis that decomposed cellulose. The main sets of microbiological compositions in the gut included Spirochaetes, Bacteroidetes, Proteobacteria, Fibrobacteres, and Actinobacteria. M. shangchengensis, which show intricate anatomical features, mainly depend on its own digestive juice secreted to decompose lignocellulose. The diets were chewed up into granules and delivered into the P3 region during preliminary digestion. The Spirochete and Fibrobacteres species in the P3 region decomposed non-starch polysaccharides, and then produced intermediates. Next, $\mathrm{O}_{2}, \mathrm{H}_{2}$, and $\mathrm{N}_{2}$ can be transformed into acetic acid and microbial proteins by $\mathrm{H}_{2}$ metabolism, $\mathrm{CO}_{2}$-reductive acetogenesis, and $\mathrm{N}_{2}$ fixation (Warnecke et al., 2007). This indicates that the mechanisms of lignocellulose degradation in the 2 types of termites were quite different.

In conclusion, the results of this study showed that the higher termite $M$. shangchengensis constituted $25 \%$ of the phylotypes, which was higher than the lower termite T. ampliceps. Without symbiotic protozoa, higher termites depended on a more complicated survival mechanism of lignocellulose degradation than did T. ampliceps. Significant impacts on the microbiota composition in the groups of $T$. ampliceps following 2-week diet shifts were observed. Our results showed that Bacteroidetes and Spirochaetes acted on celluloses, while Firmicutes was responsible for lignin degradation. Additionally, Proteobacteria was consistently involved in energy production and balancing of the gut environment. Bacteroidetes may function without hindgut protozoans in higher termites.

\section{Conflicts of interest}

The authors declare no conflict of interest.

\section{ACKNOWLEDGMENTS}

Research supported by the National Science Foundation of China (\#31170350).

\section{REFERENCES}

Berlanga M, Paster BJ, Grandcolas P and Guerrero R (2011). Comparison of the gut microbiota from soldier and worker castes of the termite Reticulitermes grassei. Int. Microbiol. 14: 83-93.

Boucher I, Vadeboncoeur C and Moineau S (2003). Characterization of genes involved in the metabolism of alpha-galactosides by Lactococcus raffinolactis. Appl. Environ. Microbiol. 69: 4049-4056.

Boucias DG, Cai Y, Sun Y, Lietze VU, et al. (2013). The hindgut lumen prokaryotic microbiota of the termite Reticulitermes flavipes and its responses to dietary lignocellulose composition. Mol. Ecol. 22: 1836-1853.

Brosius J, Palmer ML, Kennedy PJ and Noller HF (1978). Complete nucleotide sequence of a 16S ribosomal RNA gene from Escherichia coli. Proc. Natl. Acad. Sci. U. S. A. 75: 4801-4805.

Brumm P, Mead D, Boyum J, Drinkwater C, et al. (2011). Functional annotation of Fibrobacter succinogenes S85 carbohydrate active enzymes. Appl. Biochem. Biotechnol. 163: 649-657.

Brune A (2014). Symbiotic digestion of lignocellulose in termite guts. Nat. Rev. Microbiol. 12: 168-180.

Chen W, Shi Y, Peng JX, Hong HZ, et al. (2011). Phylogenetic diversity analysis and in situ hybridization of symbiotic Oxymonad 
flagellates in the hindgut of Reticulitermes chinensis Snyder. Acta Ecol. Sin. 31: 5332-5340.

Cleveland LR and Grimstone AV (1964). The fine structure of the flagellate Mixotricha paradoxa and its associated microorganisms. Proc. Roy. Soc. B Biol. Sci. 159: 668-686.

Dolan MF (2001). Speciation of termite gut protists: the role of bacterial symbionts. Int. Microbiol. 4: 203-208.

Engelbrektson A, Kunin V, Wrighton KC, Zvenigorodsky N, et al. (2010). Experimental factors affecting PCR-based estimates of microbial species richness and evenness. ISME J. 4: 642-647.

Hongoh Y, Ohkuma M and Kudo T (2003). Molecular analysis of bacterial microbiota in the gut of the termite Reticulitermes speratus. FEMS Microbiol. Ecol. 44: 231-242.

Hongoh Y, Deevong P, Inoue T, Moriya S, et al. (2005). Intra- and interspecific comparisons of bacterial diversity and community structure support coevolution of gut microbiota and termite host. Appl. Environ. Microbiol. 71: 6590-6599.

Hongoh Y, Deevong P, Hattori S, Inoue T, et al. (2006). Phylogenetic diversity, localization, and cell morphologies of members of the candidate phylum TG3 and a subphylum in the phylum Fibrobacteres, recently discovered bacterial groups dominant in termite guts. Appl. Environ. Microbiol. 72: 6780-6788.

Huang XF, Bakker MG, Judd TM, Reardon KF, et al. (2013). Variations in diversity and richness of gut bacterial communities of termites (Reticulitermes flavipes) fed with grassy and woody plant substrates. Microb. Ecol. 65: 531-536.

Husseneder C, Ho HY and Blackwell M (2010). Comparison of the bacterial symbiont composition of the Formosan subterranean termite from its native and introduced range. Open Microbiol. J. 4: 53-66.

lida T, Ohkuma M, Ohtoko K and Kudo T (2000). Symbiotic spirochetes in the termite hindgut: phylogenetic identification of ectosymbiotic spirochetes of oxymonad protists. FEMS Microbiol. Ecol. 34: 17-26.

Jiao NZ, Feng FY and Wei B (2006). Proteobacteria rhodopsin - new ways of bioavailability of marine light. Chin. Sci. Bull. 51: 887-894.

Leadbetter JR and Breznak JA (1996). Physiological ecology of Methanobrevibacter cuticularis sp. nov. and Methanobrevibacter curvatus sp. nov., isolated from the hindgut of the termite Reticulitermes flavipes. Appl. Environ. Microbiol. 62: 3620-3631.

Mathew GM, Ju YM, Lai CY, Mathew DC, et al. (2012). Microbial community analysis in the termite gut and fungus comb of Odontotermes formosanus: the implication of Bacillus as mutualists. FEMS Microbiol. Ecol. 79: 504-517.

Matson EG, Zhang X and Leadbetter JR (2010). Selenium controls transcription of paralogous formate dehydrogenase genes in the termite gut acetogen, Treponema primitia. Environ. Microbiol. 12: 2245-2258.

Noda M, Kettenmann H and Wada K (2006). Anti-inflammatory effects of kinins via microglia in the central nervous system. Biol. Chem. 387: 167-171.

Ohkuma M (2008). Symbioses of flagellates and prokaryotes in the gut of lower termites. Trends Microbiol. 16: 345-352.

Ohkuma M, Sato T, Noda S, Ui S, et al. (2007). The candidate phylum 'Termite Group 1' of bacteria, phylogenetic diversity, distribution, and endosymbiont members of various gut flagellated protists. FEMS Microbiol. Ecol. 60: 467-476.

Peng H, Gao Y and Xiao Y (2008). The high ethanol tolerance in a thermophilic bacterium Anoxybacillus sp. WP06. Sheng Wu Gong Cheng Xue Bao 24: 1117-1120.

Raychoudhury R, Sen R, Cai Y, Sun Y, et al. (2013). Comparative metatranscriptomic signatures of wood and paper feeding in the gut of the termite Reticulitermes flavipes. Insect Mol. Biol. 22: 155-171.

Scharf ME, Karl ZJ, Sethi A and Boucias DG (2011). Multiple levels of synergistic collaboration in termite lignocellulose digestion. PLoS One 6: e21709.

Schultz JE and Breznak JA (1978). Heterotrophic bacteria present in hindguts of wood-eating termites [Reticulitermes flavipes (Kollar)]. Appl. Environ. Microbiol. 35: 930-936.

Stingl U, Radek R, Yang H and Brune A (2005). "Endomicrobia": Cytoplasmic symbionts of termite gut protozoa form a separate phylum of prokaryotes. Appl. Environ. Microbiol. 71: 1473-1479.

Su LJ, Chen M, Liu GJ, Liu H, et al. (2011). Anatomic studies on digestive, reproductive system of Tsaitermes ampliceps and comparing with Odontotermes formosanus. Chin. Bull. Entomol. 48: 1024-1032.

Tan ZY, Peng GX, Xu PZ, Ai SY, et al. (2009). Diversity and high nitrogenase activity of endophytic diazotrophs isolated from Oryza rufipogon Griff. Chin. Sci. Bull. 54: 2839-2848.

Tanaka H, Aoyagi H, Shina S, Dodo Y, et al. (2006). Influence of the diet components on the symbiotic microorganisms community in hindgut of Coptotermes formosanus Shiraki. Appl. Microbiol. Biotechnol. 71: 907-917.

Warnecke F, Luginbuhl P, Ivanova N, Ghassemian M, et al. (2007). Metagenomic and functional analysis of hindgut microbiota of a wood-feeding higher termite. Nature 450: 560-565.

Yang H, Schmitt-Wagner D, Stingl U and Brune A (2005). Niche heterogeneity determines bacterial community structure in the termite gut (Reticulitermes santonensis). Environ. Microbiol. 7: 916-932.

Zoetendal EG, Akkermans AD and De Vos WM (1998). Temperature gradient gel electrophoresis analysis of $16 \mathrm{~S}$ rRNA from human fecal samples reveals stable and host-specific communities of active bacteria. Appl. Environ. Microbiol. 64: 38543859. 